\title{
Impact of the Third Dimension on EBSD Characterizations of Residual Strain
}

\author{
Stuart I. Wright and Matthew M. Nowell
}

EDAX-TSL, 392 E 12300 S, Draper, UT 84020

Electron backscatter diffraction (EBSD) has been used to characterize the distribution of residual strain in polycrystalline samples. There are two indicators of residual plastic strain in maps constructed from automated EBSD scan measurements (or Orientation Imaging Microscopy - OIM). One of these is based on the quality EBSD patterns. If the diffraction volume contains dislocations, the overall quality of the resulting EBSD pattern is diminished. The dislocations create low angle rotations of the crystal lattice relative to the original lattice orientation. Thus, the diffracting volume essentially contains multiple crystal lattices leading to superposed patterns. OIM maps based on the quality of the diffraction patterns may provide contrast based on residual strain [1].

As EBSD is particularly adept at measuring crystallographic orientation, a second method for characterizing residual strain can be achieved through investigation of low angle misorientations [26]. The low angle rotations that arise from the presence of dislocations in the microstructure not only manifest themselves in lower quality diffraction patterns but in low angle misorientations between neighboring points in the OIM scans. This approach offers more quantitative results and is generally preferred. Some attempts have been made to calibrate low angle misorientation density to quantitative measures of strain [4]. This is done essentially by calibrating the average of the density of low angle misorientations to the overall bulk strain of the material. The density of the low angle misorientations within a single grain can then be calculated by assuming a linear scaling. One assumption of such an approach is that the low angle misorientations within a grain can be determined from the orientations measured within the grain from a single planar cross-section of the grain. This would preclude the notion that dislocations would produce local lattice rotations that vary in different directions within a single grain.

In order to test this assumption, a three dimensional dataset [7-8] from a nickel based super alloy was investigated. The orientation spread for each grain is shown in figure 1a. The deviation from the average orientation for each individual grain is shown in figure $1 \mathrm{~b}$. The maps in figure 1 were constructed from an OIM scan near the center of the overall volume characterized. The orientation spread in $x, y$ and $z$ cross sections was calculated for two different grains- one exhibiting a fair amount of orientation spread and a second exhibiting very little spread. There is clearly a significant anisotropy in the spread in the first grain. The spread in the second grain may simply be noise in the orientation measurements. While the anisotropy observed is likely a function of stress-state it should be noted that the local stress-state is likely to vary locally.

\section{References}

[1] S. I. Wright and M. M. Nowell, Microscopy \& Microanalysis, 12 (2006) 72.

[2] S. I. Wright, J. Computer Assisted Microscopy, 5 (1993) 207.

[3] S. I. Wright, D. P. Field and D. J. Dingley, Electron Backscatter Diffraction in Materials Science, Kluwer Academic/Plenum Publishers, New York (2000) 141.

[4] E. M. Lehockey, Y.-P. Lin and O. E. Lepik, Electron Backscatter Diffraction in Materials Science, Kluwer Academic/Plenum Publishers, New York (2000) 247. 
[5] B. L. Li, A. Godfrey and Q. Liu, Materials Science Forum, 408-412 (2002) 1185.

[6] L.N. Brewer, M.A. Othon, L.M. Young and T.M. Angeliu, Microscopy \& Microanalysis, 12 (2006) 85.

[7] M. D. Uchic, M. Groeber, R. Wheeler IV, F. Scheltens and D. M. Dimiduk: Microsc. Microanal.

Vol. 10 (Suppl 2) (2004), p. 1136CD.

[8] M. Uchic at Wright-Patterson Air Force Base is acknowledged for providing the authors access to the three-dimensional OIM data.
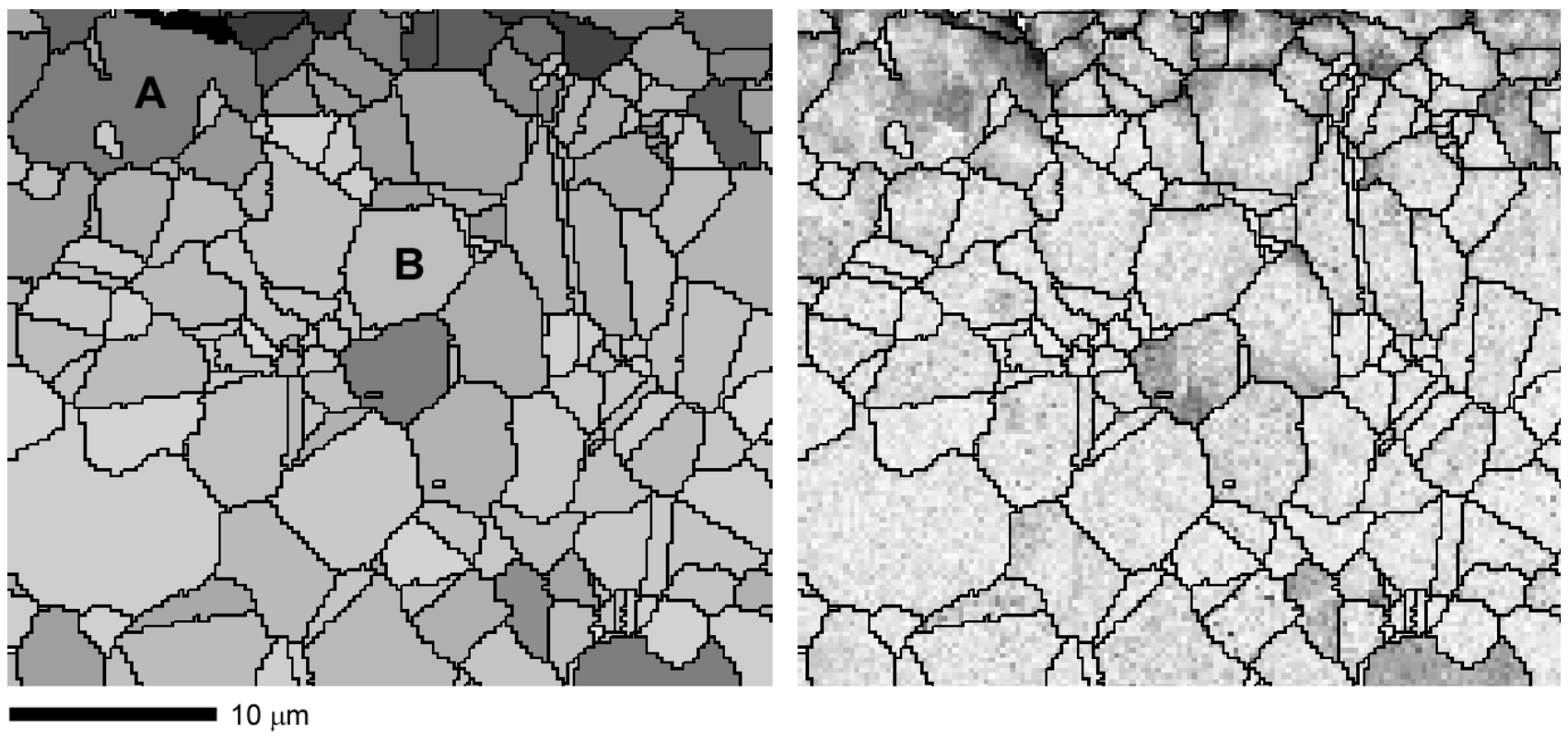

Fig. 1. (a) OIM map showing the orientation spread (the gray scale spans from white equal to $0^{\circ}$ spread to black indicating $3.3^{\circ}$ spread). (b) OIM map showing the deviation in orientation from the average orientation within individual grains (the gray scale spans from white equal to $0^{\circ} \mathrm{spread}$ to black indicating $4^{\circ}$ spread - the maximum measured was $6.8^{\circ}$ ).
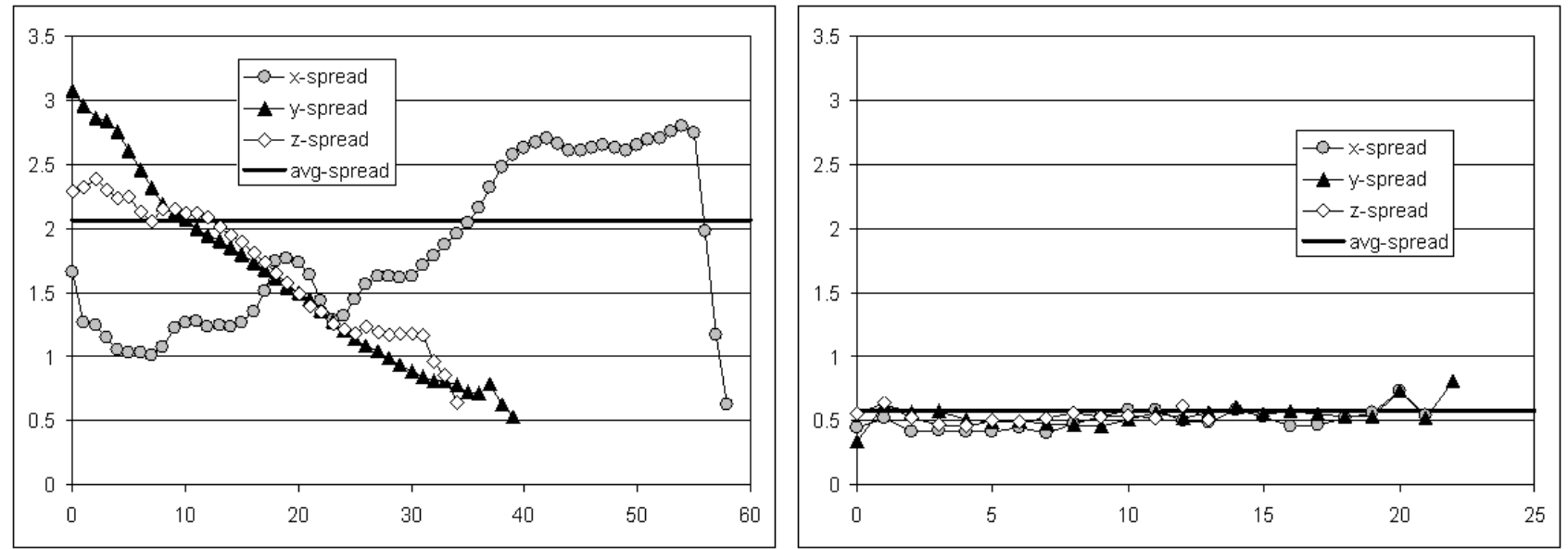

Fig. 2. Orientation spreads as a function of direction for grains " $\mathrm{A}$ " and :B" as labeled in figure 1. $x$ is the horizontal direction, $y$ - the vertical direction and $z$ - a direction normal to the page. 